\section{Reproducibility of blood flow velocity measurements using colour decoded Doppler imaging}

ET Matthiessen, O Zeitz, G Richard and M Klemm E.T. Matthiessen, none; O. Zeitz, none; G. Richard, none; M. Klemm, none.
Commercial relationship:

\begin{abstract}
Keywords: colour decoded Doppler imaging; ocular perfusion; reproducibility; glaucoma

\section{Introduction}

Colour Doppler imaging (CDI) has numerous potential applications in the diagnosis of different arterial and venous disorders in many medical specialities. Its main indications are the evaluation of vascular disorders. ${ }^{1}$ Owing to advances in sonographic imaging in the last decades, which produce real-time information on the blood velocity of even small vessels, CDI has been introduced into ophthalmology as a new method to investigate ocular blood velocity. The colour decoded Doppler imaging allows a selective and noninvasive visualization and measurement of various haemodynamic parameters of the retrobulbar vessels that maintain the eye with blood. ${ }^{2}$

The disturbance of retrobulbar haemodynamics plays a prominent role in the pathophysiology of several ocular diseases. This includes vascular processes like the occlusion of the central retinal artery, central retinal vein or their branches as well as acute occlusion of choroidal vessels leading to acute ischaemia of the optic nerve (acute ischaemic posterior optic neuropathy). ${ }^{3}$ Diminished ocular perfusion is also discussed to contribute to the progression of several forms of open-angle glaucoma. ${ }^{4-6,8}$ Glaucoma-related atrophy of the optic nerve head is thought to be primarily caused by an increased intraocular pressure (IOP). ${ }^{9}$

Nevertheless, some patients with normal IOP develop similar glaucomatous disc and visual field changes, ${ }^{6,2}$ which indicates that other risk factors must be involved in the pathogenesis of the disease.

Numerous studies using CDI have evaluated ocular haemodynamics in patients with primary open-angle glaucoma (POAG) and normal pressure glaucoma (NPG) vs age-matched

practice and research.

Eye (2004) 18, 400-405. doi:10.1038/sj.eye.6700651

Conclusions The ICCs of the repeated measurements reflect a good reproducibility for both the groups with assumed different retrobulbar perfusion. These findings are prerequisites for the use of CDI in clinica

Background It is taken for granted that
glaucomatous damage is caused by changed haemodynamics of the retrobulbar vessel system besides other factors such as, for This was proven by various studies in whic coma patients were shown to have a this study, the reliability of measurement retrobulbar vessel perfusion by colour evaluated. volunteers and 15 patients with various study. Using a CDI system, type Siemens Sonoline Elegra ${ }^{\circledR}$ with a combined perfusions of the ophthalmic artery, the long posterior ciliary arteries of each patient were measured six times. In end-diastolic velocity, maximum systolic index of the vessels were determined. evaluated by the calculation of the for each parameter.

Results The ICCs for the ophthalmic artery ciliary artery from 0.75 to $\mathbf{0 . 9 1}$, and for the long posterior ciliary artery from 0.77 to 0.99 in both
\end{abstract} Accepted in revised form ;

Universitätsklinikum Kamburg-Eppendorf Augenheilkunde
Hamburg, Germany ET Matthiessen Hamburg-Eppendorf Klinik und Poliklinik für Martinistraße 52 D-20246 Hamburg, Germany Fax: + 49040428033801 E-mail e.matthiessen@

uke.uni-hamburg.de

Received: 9 October 2002 6 May 2003 
healthy volunteers. ${ }^{10-13,2}$ In essence, the reports could demonstrate an altered retrobulbar blood flow velocity in glaucoma.

Nevertheless, the study results are partially contradictory. While Klingmüller et $a l^{2}$ could not find differences in the blood flow velocities through the short posterior ciliary artery between patients with POAG and healthy age-matched controls, Kaiser et al ${ }^{12}$ could demonstrate significant differences.

In subsumption, some reports do not confirm reports on an altered retrobulbar blood flow velocity in glaucoma patients. Also, researchers question the reproducibility of the CDI measurements. ${ }^{14-16}$ Unfortunately, despite the various studies using CDI for measuring ocular blood velocity, no assessment of reproducibility measurements has been carried out for the ophthalmic artery (OA), the short posterior ciliary artery (SPCA), and the long posterior ciliary artery (LPCA).

The purpose of this study is to investigate the reproducibility of retrobulbar blood flow velocity measurements. For the application of this method in glaucoma patients, it is extremely important to know whether the measured values are reproducible, that is, whether an evaluation of the haemodynamics by CDI is feasible. This is tested in two collectives: one healthy and one whose retrobulbar haemodynamics is assumed to be impaired.

\section{Materials and methods}

\section{Study group}

A total of 33 subjects (18 healthy volunteers and 15 patients with treated or untreated glaucoma) were enrolled in this study. None of the healthy volunteers had any significant cardiovascular, respiratory, neurologic, or ophthalmic history. They all had normal clinical eye examination results (visus, slit-lamp examination of the anterior segment, intraocular tension, optic disc assessment using indirect ophthalmoscopy with a $90 \mathrm{D}$ lens). Volunteers using any systemic medication were excluded. The patients of the second group had NPG or POAG with characteristic optic disc changes and visual field defects. All patients were in-patients at the University Eye Clinic, Hamburg (Germany). One randomly chosen eye in each individual was studied.

All patients and volunteers provided informed consent for study participation. The study protocol was approved by the local ethics committee.

\section{Colour Doppler imaging}

CDI was performed with a Sonoline Elegra Advanced System (Siemens, Erlangen, Germany) using a phased array transducer type 7.5L40 (Siemens, Erlangen, Germany). Ultrasound frequency was $6.5 \mathrm{MHz}$ in the pulsed Doppler mode. The examination was conducted with the patient in a supine position and the head elevated at a $30^{\circ}$ angle. The ocular examination lasted approximately $30-40 \mathrm{~min}$. The transducer was carefully set on the closed eye lids without exerting pressure on the bulb. Acoustic coupling between transducer and skin was optimized by a carbomeric gel (Vidisic ${ }^{\circledR}$, Dr Mann Pharma, Germany). In the first instance, a B-scan of the optic nerve was obtained, which provides the most useful landmark for the identification of the retrobulbar vessels. The OA, which is a branch of the internal carotid artery, is situated above or below the optic nerve in the posterior orbit and passes forward into the nasal orbit. After crossing the optic nerve, the OA could be traced through the flow towards the applicator and by the typical pulsatility approximately $15 \mathrm{~mm}$ posterior to the globe in every individual. ${ }^{17}$ The ciliary arteries can be classified into three groups: the long and short posterior, as well as the anterior ciliary arteries. The short posterior ciliary arteries from six to 12 in number arise from the OA or its branches. They begin as trunks approximately $10-20 \mathrm{~mm}$ behind the globe, before they form multiple branches surrounding the optic nerve in its retrobulbar portion. They pierce the sclera around the optic nerve and supply the choroid and ciliary processes. Because of the high variability of the course, we choose the first point behind the globe where they could be measured and show characteristic Doppler spectra as the point to get the best reproducibility. The two LPCAs could be localized more distal from the optic nerve and were measured directly behind the sclera before their entry into the sclera. They are running forward, along either side of the eyeball, between the sclera and choroids, to supply the anterior part of the eye with blood. For every individual we took the SPCA and LPCA with highest velocity. The third group of ciliary arteries, the anterior ciliary arteries, which were not measured, are derived from the muscular branches and are running to the front of the eyeball ending in the circulus arteriosus major.

The angle between the transducer and the orientation of the vessel was corrected. Gain and threshold had to be adjusted individually for each examination until noise disappeared and was kept constant during the entire examination. Pulse repetition frequency (PRF) was minimized to avoid aliasing (typically $5208 \mathrm{~Hz}$ for the OA and $2500 \mathrm{~Hz}$ for the SPCA and LPCA in the Doppler mode). The sample size for all vessels was set constantly to $1.5 \mathrm{~mm}$.

From the continuous recording of velocities over time, peak systolic velocity (PSV) and end diastolic velocity (EDV) were taken. The time average maximum velocity (TAMx) was calculated as well as the pulsatility index 
(PI) and the resistive index (RI). ${ }^{7}$

$$
\mathrm{RI}=\frac{(\text { peak systolic velocity })-(\text { end diastolic velocity })}{\text { peak systolic velocity }}
$$

\section{Statistical analysis}

In this study, the examination of each of the three vessels was repeated six times during a single session. After each single measurement of one vessel, the investigator had to take the applicator away from the eye and had to make a complete new measurement like examining a new patient. To avoid the examiner being affected by the previous measurement, he had to change the vessel after each single measurement. The investigation of a patient comprised six rounds of a series of measurements. The analysis of the blood flow velocity parameters took place following the investigation. This procedure was necessary to test the reproducibility of the measurements. All numerical computations were performed using SPSS software (release 10.0 for Windows $\left.{ }^{\circledR}\right)$. The results were expressed as mean \pm standard deviation (SD) for each group. The reproducibility measurements were evaluated by calculating the intraclass correlation coefficient (ICC). The ICC as an index of reliability is used to measure the extent to which an item, scale, or instrument will yield the same score when administered in different times, locations, or populations, when the two administrations do not differ in relevant variables. The reliability is calculated from the natural variance of the true value and the variance in the error in measurements. If the variance of the error in measurements is small, the reliability will have a high value. The closer the ICC to 1.0, the more reliable the method. ${ }^{18}$ The ICC was calculated for each evaluated vessel (OA, SPCA, and LPCA) separately for every parameter (PSV, EDV, RI and PI). The ICCs displayed in the result section were calculated by forming the means of each group.

\section{Results}

The analysis was based on 18 healthy volunteers and 15 patients with NPG or POAG.

The OA, SPCA, and LPCA were visualized and evaluated in all individuals. The OA could be easily detected by the typical flow velocity waveform. The waveform shows a high systolic peak flow velocity at the beginning of the cardiac cycle, a fast decrease of the velocity afterwards, and a good visible velocity at the ending of the cycle. Figures 1a-c show the OA and the flow velocity profile of one healthy volunteer at three different times. As described before, for every new
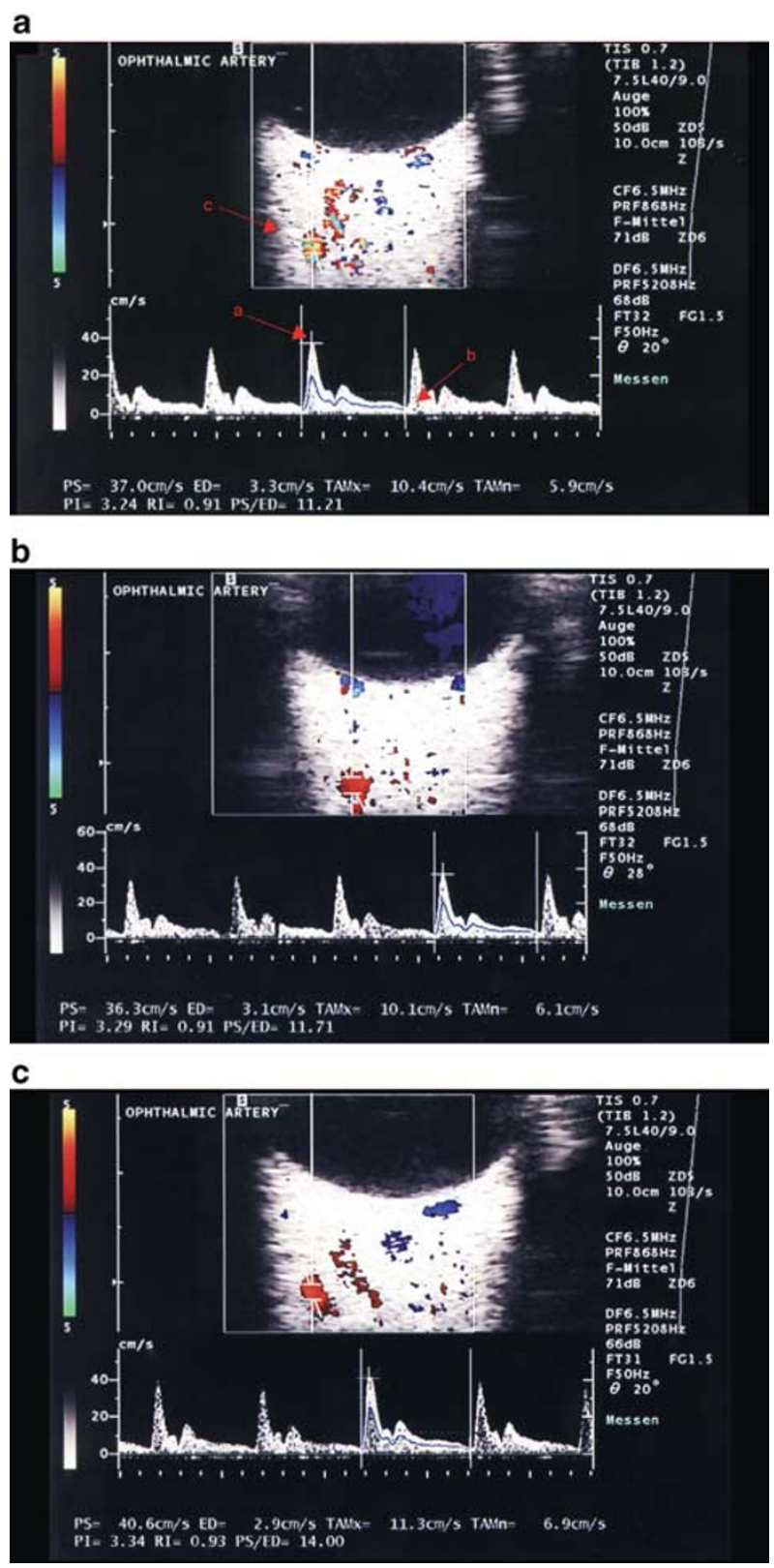

Figure $1(\mathrm{a}-\mathrm{c})$ Three horizontal scans through the globe showing the ophthalmic artery of one healthy volunteer at different times. The OA can be traced after its crossing the optic nerve slightly medial to the optic nerve. The waveform of the flow velocity shows a high maximum systolic peak (a) and a good visible peak-end diastolic velocity (b) The ophthalmic artery is marked with c.

measurement of one vessel, the setup had to be changed again from the beginning. The angle correction of the sample size is different in all three figures to be exactly aligned to the course of the vessel.

The SPCA could easily be detected beside the optic nerve directly behind the sclera. The maximum systolic peak velocity is three to four times smaller in comparison 


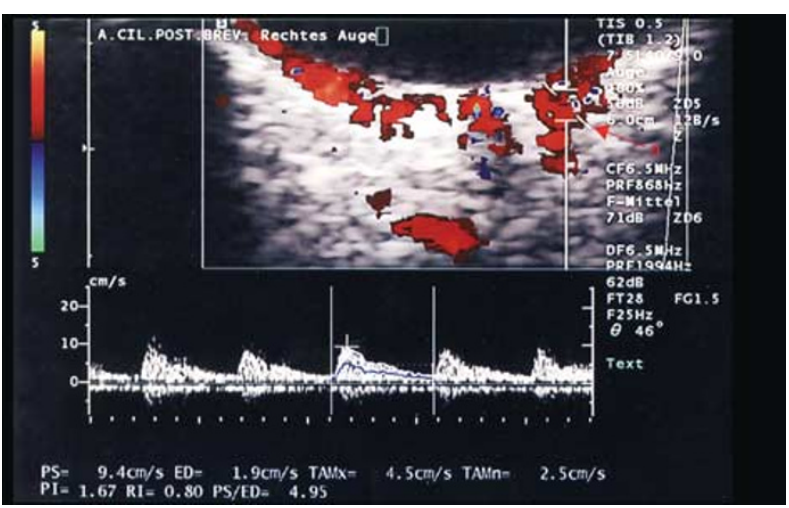

Figure 2 Horizontal scans through the globe showing the SPCAs of a healthy volunteer. There is an apparent maximum systolic peak velocity and a very low maximum peak-end diastolic velocity. $\mathrm{a}=$ angle correction.



Figure 3 Horizontal scans through the globe showing the LPCAs of a healthy volunteer. There is an apparent maximum systolic peak velocity and a very low peak-end diastolic velocity. $\mathrm{a}=$ angle correction.

to that of $\mathrm{OA}$ and the pronounced decrease after the peak observed in the OA is missing (Figure 2). The LPCA is beyond the SPCA and measured directly behind the sclera before their entry into the sclera (Figure 3). In the lower part of Figures 1-3, the Doppler spectrum (velocity-time curve) can be seen. The SPCA shows a similar velocity as the LPCA. The pronounced aortic valve incision at the end of the systole observed in the OA is still there but less pronounced in the SPCA and LPCA. Using cursors, analysis of the spectrum and measurement of the flow velocities are performed. The calculated parameters are illustrated at the bottom (Figures 1-3).

Blood flow velocities, the RIs, and PIs of the measured vessels are displayed in Table 1 . The mean values of both groups were similar regarding all variables. A direct statistic comparison of the values was not performed because of the high heterogeneity of the groups.
Table 1 Descriptive statistics of the measured variables

\begin{tabular}{lcc}
\hline Variable & \multicolumn{2}{c}{ Mean and standard deviation $\left(\mathrm{cm} \mathrm{s}^{-1}\right)$} \\
\cline { 2 - 3 } & Healthy volunteers & Glaucoma patients \\
\hline OP-PSV & $32.40 \pm 4.34$ & $27.56 \pm 4.47$ \\
SPCA-PSV & $8.44 \pm 1.36$ & $7.58 \pm 0.81$ \\
LPCA-PSV & $13.27 \pm 3.64$ & $8.75 \pm 1.67$ \\
OA-ED & $7.31 \pm 2.67$ & $4.36 \pm 1.18$ \\
SPCA-ED & $2.62 \pm 0.32$ & $2.06 \pm 0.29$ \\
LPCA-ED & $3.13 \pm 0.87$ & $2.14 \pm 0.42$ \\
OA-PI & $2.01 \pm 0.36$ & $2.46 \pm 0.55$ \\
SPCA-PI & $1.36 \pm 0.17$ & $1.56 \pm 0.16$ \\
LPCA-PI & $1.88 \pm 0.32$ & $1.61 \pm 0.15$ \\
PA-RI & $0.77 \pm 0.06$ & $0.95 \pm 0.24$ \\
SPCA-RI & $0.68 \pm 0.04$ & $0.72 \pm 0.02$ \\
LPCA-RI & $0.75 \pm 0.03$ & $0.74 \pm 0.03$ \\
\hline
\end{tabular}

Healthy volunteers, $n=18$; glaucoma patients, $n=15$. PSV, peak systolic velocity; ED, end diastolic velocity; PI, pulsatility index; RI, resistivity index.

Table 2 ICC of every determined variable for the OA, SPCA, and LPCA

\begin{tabular}{lcccc}
\hline Vessel & \multicolumn{4}{c}{ Intraclass correlation coefficient } \\
\cline { 2 - 5 } & $P S\left(\mathrm{~cm} \mathrm{~s}^{-1}\right)$ & $E D\left(\mathrm{~cm} \mathrm{~s}^{-1}\right)$ & $P I$ & $R I$ \\
\hline Healthy volunteers & & & & \\
OA & 0.98 & 0.92 & 0.97 & 0.92 \\
SPCA & 0.88 & 0.85 & 0.83 & 0.71 \\
LPCA & 0.99 & 0.93 & 0.92 & 0.77 \\
Glaucoma patients & & & & \\
OA & 0.9 & 0.89 & 0.93 & 0.90 \\
SPCA & 0.91 & 0.75 & 0.82 & 0.78 \\
LPCA & 0.96 & 0.93 & 0.87 & 0.84 \\
\hline
\end{tabular}

The reproducibility measurements were evaluated by the calculation of the ICC for every variable in each vessel. The variation within the observer was low for all four parameters in the three vessels, corresponding to ICCs displayed in Table 2. The displayed ICCs represented the reproducibility of measurements in each group. The highest reliability could be achieved for the PSV followed by the ICC for the EDV in all vessels of both groups (Table 2).

\section{Discussion}

The aim of this study was to evaluate whether the CDI is a reliable method for the measurement of blood flow velocity in retrobulbar vessels. This was tested in healthy volunteers with assumed normal retrobulbar blood flow velocity, as well as in glaucoma patients with assumed impaired retrobulbar blood flow velocity. Our results 
show that the reproducibility of the CDI measurements is not affected by the quantity of the blood circulation.

Although the CDI has been used in many studies to examine the retrobulbar blood flow velocities, some authors have pointed out that the reliability of this method is poor. For repeated measurements, especially in follow-up examinations, however, a high reliability is necessary. Recently, Quaranta et $a l^{19}$ demonstrated a low variability of OA measurements performed by different raters. They concluded that CDI may be a useful tool to study the blood flow velocity in the OA. Other studies could find reliable values for the OA, the nasal short posterior ciliary artery, (NSPCA) the temporal short posterior ciliary artery (TSPCA), and the central retinal artery (CRA). ${ }^{14}$ They performed test/retest measurements by the same operator within one session and achieved the highest reproducibility for the OA. In this study, we performed likewise test/retest measurements. To mask the observer from the previous findings of the previous examination, the examiner had to switch between the vessels after each single measurement. Thus, the configuration of the CDI had to be newly configured for each measurement separately. Owing to this procedure, the number of measured vessels had to be limited to avoid too long an investigation duration. In addition, the analysis of the parameters took place only after completing all measurements. We decided on this kind of masking, because other methods of masking, like repeated examinations on different days, would result in a higher variability caused by other factors with potential effects on retrobulbar blood flow velocity. For example, natural fluctuations of the blood pressure or intraocular pressure could lead to a higher variability of the retrobulbar blood flow velocity, making an analysis of reproducibility senseless.

Our results confirm the findings of the other studies in which the highest reproducibility was achieved for the OA. However, there is still the view that measurements of the smaller vessels are more variable and therefore the CDI is not sufficiently reliable for the assessment of these vessels. Especially referring to glaucomatous optic neuropathy, it is interesting to know if the SPCA can be reliably measured. The SPCA supplies the optic nerve head, the area of direct glaucomatous damage, with blood. For clinicians, it would be very important to know whether the blood flow velocity of the ciliary arteries is reduced or not. Our results show that also for smaller vessels highly reliable coefficients of CDI measurements can be achieved. Even in the SPCA, whose diameter is obviously smaller than that of the OA, we could achieve an ICC of 0.88 for healthy volunteers and 0.91 for the affected group referring the PSV. A value above 0.7 reflects a reliable measurement. ${ }^{18}$ We found ICCs above 0.7 in all measured parameters and in both groups, that is, also in the group of glaucoma patients whose blood flow velocity is supposed to be affected. This suggests that the reliability does not depend on the degree of blood flow velocity. Possibly the high reproducibility can be explained by the strict test situation. All patients had the same seating and head position.

Since the resistance indices are a ratio of velocities and are angle independent, they are supposed to be more reliable parameters than flow velocities within the small vessels. This study shows no differences in the reliability of the different variables. Thus, the CDI allows a differentiated determination of blood flow velocities. However, the interpretation of the measured values remains difficult. CDI measures blood flow velocities and not blood flow because of the missing determination of the accurate diameter of orbital vessels with this technique. However, other studies could demonstrate a good correlation between blood flow velocity and blood flow, especially in cerebral vessels, ${ }^{20,21}$ as well as partial correlations with other methods measuring ocular haemodynamics. ${ }^{22}$

The results of our study suggest that the basic condition for clinical application - a good reproducibility of CDI measurements - is given. This should allow one to differentiate between normal and abnormal, even when the measurements are performed by different examiners, for example, throughout the follow-up.

In summary, our investigations supply the evidence that CDI is a suitable method to examine retrobulbar blood flow velocities in clinical practice and research. Moreover, the differentiated determination of retrobulbar blood velocities might lead to a better understanding of the pathophysiology of ocular diseases with disturbed ocular haemodynamics.

\section{References}

1 Scout LM, Zawin KJ, Taylor J, Doppler US. II. Clinical applications. Radiology 1990; 174: 309-319.

2 Klingmüller VK, Schmidt KG, Rückmann Av, Koch B, Stein A. Doppler-sonography of the short posterior ciliary arteries in primary open angle glaucoma patients. Ultraschall Med 2000; 21(1): 32-37.

3 Ghanchi F D, Williamson TH, Lim CS, Butt Z, Baxter GM, McKillop $G$ et al. Colour Doppler imaging in giant cell (temporal) arteritis: serial examination and comparison with non-arteritic anterior ischaemic optic neuropathy. Eye 1996; 10(Part 4): 459-464.

4 Hayreh S, Revie I, Edwards J. Vasogenic origin of visual field defects and optic nerve changes in glaucoma. $\mathrm{Br} J$ Ophthalmol (England) 1970; 54(7): 461-472.

5 Maumenee A. Causes of optic nerve damage in glaucoma. Robert N. Shaffer lecture. Ophthalmology 1983; 90(7): 741-752.

6 Pillunat L, Stodtmeister R, Marquardt R, Mattern A. Ocular perfusion pressures in different types of glaucoma. Int Ophthalmol 1989; 13(1-2): 37-42. 
7 Pourcelot L. Applications cliniques de l'examen Doppler transcutané. Inserm 1974; 84: 213-240.

8 Schmidt KG, Rückmann AV, Mittag TW, Hessemer V, Pillunet LE. Reduced ocular pulse amplitude in low tension glaucoma is independent of vasospasm. Eye 1997; 11(Part 4): 485-488.

9 Goldmann H. Open-angle glaucoma. Br J Ophthalmol 1972; 56: 242-248.

10 Butt Z, McKillop G, O'Brien C, Allan P, Aspinall P. Measurement of ocular blood flow velocity using colour Doppler imaging in low tension glaucoma. Eye 1995; 9(Part 1): 29-33.

11 Butt Z, O'Brien C, McKillop G, Aspinall P, Allan P. Color Doppler imaging in untreated high and normal pressure open-angle glaucoma. Invest Ophthalmol Vis Sci 1997; 38(3): 690-696.

12 Kaiser H, Schoetzau A, Stumpfig D, Flammer J. Blood flow velocities of the extraocular vessels in patients with high tension and normal tension primary open-angle glaucoma. Am J Ophthalmol 1997; 123(3): 320-327.

13 Liu C, Chou Y, Chou J, Chou J, Chiou H, Chiang S et al. Retrobulbar haemodynamic changes studied by colour Doppler imaging in glaucoma. Eye 1997; 11(Part 6): 818-826.

14 Baxter G, Williamson T. Color Doppler imaging of the eye: normal ranges, reproducibility, and observer variation. J Ultrasound Med 1995; 14(2): 91-96.
15 Harris A, Williamson T, Martin B, Shoemaker J, Sergott S, Spaeth RG et al. Test/retest reproducibility of colour Doppler imaging assessment of blood flow velocity in orbital vessels. J Glaucoma 1995; 4: 281-286.

16 Williamson T, Harris A. Color Doppler ultrasound imaging of the eye and orbit. Surv Ophthalmol (United States) 1996; 40(4): 255-267.

17 Lieb W. Color Doppler imaging of the eye and orbit. Radiol Clin North Am 1998; 36(6): 1059-1071.

18 Shrout PE, Fleiss JL. Intraclass correlations: uses in assessing rater reliability. Psychol Bull 1979; 86: 420-428.

19 Quaranta L, Harris A, Donato F, Cassamali M, Semeraro F, Nascimbeni G et al. Color Doppler imaging of ophthalmic artery blood flow velocity: a study of repeatability and agreement. Ophthalmology 1997; 104(4): 653-658.

20 Hansen NB, Stonestreet BS, Rosenkrantz TS, Oh W. Validity of Doppler measurements of anterior cerebral artery blood flow velocity: correlation with brain blood flow in piglets. Pediatrics 1983; 72: 526-531.

21 Taylor GA, Short BL, Walker LK, Traystman RJ. Intracranial blood flow: quantification with duplex Doppler and color Doppler flow US. Radiology 1990; 176: 231-236.

22 Zeitz O, Matthiessen ET, Richard G, Klemm M. Estimation of choroid perfusion by colour Doppler imaging versus other methods. Ultrasound Med Biol, 2002; 28: 1023-1027. 